\title{
Peritonitis caused by Ewingella americana in a patient with peritoneal dialysis: a case report
}

\author{
Li Li, Jianxiong Shen, Jianmin Tao and Zhizhong Xue
}

\begin{abstract}
Introduction: Peritonitis can happen in patients undergoing continuous ambulatory peritoneal dialysis. It is commonly caused by Staphylococcus aureus, which is the basis of the empiric choice of antibiotics. Here we report a case that was caused by Ewingella americana and thus required different considerations of treatment.

Case presentation: A 76-year-old Chinese woman underwent continuous ambulatory peritoneal dialysis after the diagnosis of chronic renal failure. She presented with generalized abdominal pain after the start of continuous ambulatory peritoneal dialysis for 10 consecutive months. The pathogen was identified as Ewingella americana by microbiological examination and its susceptibility to antibiotics was partly different from the common pathogens causing peritonitis. The change of antibiotic treatment from vancomycin to amikacin accelerated her recovery.

Conclusion: It should be noted that peritonitis can be caused by E. americana. Our report may add to the known causes of peritonitis in a hospital environment. With respect to the empiric choice of antibiotics to treat peritonitis under the condition of continuous ambulatory peritoneal dialysis, it is worth noting that $E$. americana has a different susceptibility to the spectrum of antibiotics and its corresponding therapy should be taken into consideration.
\end{abstract}

Keywords: Peritonitis, Peritoneal dialysis, Ewingella americana

\section{Introduction}

Peritonitis is mainly caused by infection due to the disruption of the peritoneum, commonly under the condition of continuous ambulatory peritoneal dialysis (CAPD). Peritoneal dialysis (PD) is used as a treatment for patients with severe chronic renal disease. The process uses the patient's peritoneum in the abdomen as a membrane across which fluids and dissolved substances (electrolytes, urea, glucose, albumin and other small molecules) are exchanged from the blood. This regular exchange is performed by catheterization in the abdomen which increases the opportunity for infection. Peritonitis primarily caused by bacteria has more clear symptoms of infection in contrast to those subsequent to perforation of part of the gastrointestinal tract.

Ewingella americana was first described from clinical samples by Grimont et al. in 1983 [1]. It rarely causes human infections, although several investigations reported

\footnotetext{
* Correspondence: yyyylab@126.com

Yueyang Hospital of Integrated Traditional Chinese and Western Medicine, Shanghai University of Traditional Chinese Medicine, No.110, Ganhe road, Shanghai 200437, China
}

(c) 2014 Li et al.; licensee BioMed Central Ltd. This is an Open Access article distributed under the terms of the Creative Commons Attribution License (http://creativecommons.org/licenses/by/2.0), which permits unrestricted use, distribution, and reproduction in any medium, provided the original work is properly credited. The Creative Commons Public Domain Dedication waiver (http://creativecommons.org/publicdomain/zero/1.0/) applies to the data made available in this article, unless otherwise stated.

its existence in sputum [2], conjunctiva [3,4], blood [5-8], wounds [9], peritoneal dialysis [10] and bone marrow [11].

Here we report a case of peritonitis due to E. americana in a patient with end-stage renal disease undergoing CAPD.

\section{Case presentation}

A 76-year-old Chinese woman underwent CAPD after the diagnosis of chronic renal failure (uremia). Surgery for peritoneal dialysis was performed by successful catheterization under the help of local anesthesia. She was admitted due to generalized abdominal pain after a period of 10 months of CAPD. She had symptoms of decreased appetite, left chest pain and pressure, which was worse at night, but no nausea and acid regurgitation. Physical examination revealed tenderness and positive rebound.

The hematological tests revealed a hemoglobin level of $10.9 \mathrm{~g} / \mathrm{dl}$, a platelet count of $201 \times 10^{9} /$ liter, a white cell count of $11.6 \times 10^{9} /$ liter. The peritoneal dialysate was turbid, and microscopic examination showed 400 cells $/ \mathrm{mm}^{3}$, with a predominance of neutrophils. The diagnosis of peritonitis was established, and our patient was treated 
Table 1 Antimicrobial susceptibility testing of E. Americana

\begin{tabular}{lll}
\hline Antimicrobial agent & MIC $(\boldsymbol{\mu g} / \mathbf{m l})$ & Interpretation \\
\hline Ampicillin & $>=32$ & $\mathrm{R}$ \\
Ampicillin and/or Sulbactam & $>=33$ & $\mathrm{R}$ \\
Cefazolin & $>=64$ & $\mathrm{R}$ \\
Cefotetan & $>=64$ & $\mathrm{R}$ \\
Ertapenem & $>=8$ & $\mathrm{R}$ \\
Tobramycin & $<=1$ & $\mathrm{~S}$ \\
Trimethoprim and/or Sulfamethoxazole & 80 & $\mathrm{R}$ \\
Ceftazidime & $<=1$ & $\mathrm{~S}$ \\
Ceftriaxone & 16 & $\mathrm{I}$ \\
Cefepime & $<=1$ & $\mathrm{~S}$ \\
Aztreonam & 4 & $\mathrm{~S}$ \\
Imipenem & $<=1$ & $\mathrm{~S}$ \\
Amikacin & $<=2$ & $\mathrm{~S}$ \\
Gentamicin & $<=1$ & $\mathrm{~S}$ \\
Ciprofloxacin & $<=0.25$ & $\mathrm{~S}$ \\
Piperacillin and/or Tazobactam & $<=4$ & $\mathrm{~S}$ \\
Nitrofurantoin & 128 & $\mathrm{R}$ \\
Levofloxacin & 0.5 & $\mathrm{~S}$ \\
\hline
\end{tabular}

MIC, Minimal inhibitory concentration; R, Resistant; $S$, Sensitive.

empirically with vancomycin intravenously. Samples of dialysate were obtained and inoculated onto $5 \%$ sheep blood agar. After $48 \mathrm{~h}$ of incubation at $37^{\circ} \mathrm{C}$, a gram-negative, lactose-fermenting rod was observed that showed oxidase negative and catalase positive. The isolate was identified by the VITEK $^{\circ}$ compact identification systems (bioMérieux Vitek Inc., Hazelwood, MO, USA) as E. americana. E. americana was further tested by sequencing the $16 \mathrm{~s}$ ribosomal deoxyribonucleic acid (rDNA) (Sangon Biotech, Shanghai, China) and confirmed by comparison with the genetic sequence published previously.

Antimicrobial susceptibility was determined by the VITEK $^{\circ}$ compact system (using card AST-GN 13). The isolate was found to be susceptible to tobramycin, ceftazidime, cefepime, aztreonam, imipenem, amikacin, gentamicin, ciprofloxacin, piperacillin and/or tazobactam and levofloxacin but resistant to ampicillin, ampicillin and/or sulbactam, cefazolin, cefotetan, ertapenem, trimethoprim and/or sulfamethoxazole and nitrofurantoin (Table 1). After susceptibility results were obtained, vancomycin therapy was stopped and changed to amikacin until our patient's complete recovery.

\section{Conclusion}

This is a case report from Asia showing that peritonitis can be caused by E. americana. E. americana is rarely isolated, especially as the pathogen for peritonitis. Our case report may add to the causes of peritonitis in the hospital environment. Since this organism can survive in water with simple nutritional provision, hospitals' in-house sources of water, including air conditioning units, ice baths, wound cleaning devices and catheterization, can be the sources of infection.

Peritonitis is mainly caused by the disruption of the peritoneum, simply by letting micro-organisms into the peritoneal cavity, such as under the condition of CAPD. The most common isolates include cutaneous species, such as Staphylococcus aureus, and coagulase-negative staphylococci. Thus, the empiric choice of antibiotics often targets $S$. aureus (vancomycin), whereas in our case it should be noted that E. americana can also be a cause of peritonitis and its corresponding therapy should be taken into consideration.

\section{Consent}

Written informed consent was obtained from the patient for publication of this case report and accompanying images. A copy of the written consent is available for review by the Editor-in-Chief of this journal.

\section{Abbreviations}

CAPD: Continuous ambulatory peritoneal dialysis; PD: Peritoneal dialysis; MIC: Minimum inhibitory concentration.

\section{Competing interests}

The authors declare that they have no competing interests.

\section{Authors' contributions}

$\mathrm{LL}$ analyzed the case, performed the microbiological examination and was a major contributor in writing the manuscript. JXS interpreted the test results of our patient. JMT performed the laboratory tests. ZZX organized the relevant tests and analyzed our patient's results. All authors read and approved the final manuscript.

Received: 2 October 2013 Accepted: 30 December 2013

Published: 4 March 2014

\section{References}

1. Grimont PA, Farmer JR 3rd, Grimont F, Asbury MA, Brenner DJ, Deval C: Ewingella americana gen.nov., sp.nov., a new Enterobacteriaceae isolated from clinical specimens. Ann Microbiol (Paris) 1983, 134:39-52.

2. Ryoo NH, Ha JS, Jeon DS, Kim JR, Kim HC: A case of pneumonia caused by Ewingella americana in a patient with chronic renal failure. J Korean Med Sci 2005, 20:143-145.

3. Da Costa PS, Tostes MM, de Carvalho Valle LM: A case of keratoconjunctivitis due to Ewingella americana and a review of unusual organisms causing external eye infections. Braz J Infect Dis 2000, 4:262-267.

4. Heizmann WR, Michel R: Isolation of Ewingella americana from a patient with conjunctivitis. Eur J Clin Microbiol Infect Dis 1991, 10:957-959.

5. Pien FD, Farmer JR 3rd, Weaver RE: Polymicrobial bacteremia caused by Ewingella americana (family Enterobacteriaceae) and an unusual Pseudomonas species. J Clin Microbiol 1983, 18:727-729.

6. Devreese K, Claeys G, Verschraegen G: Septicemia with Ewingella americana. J Clin Microbiol 1992, 30:2746-2747.

7. Maertens J, Delforge M, Vandenberghe P, Boogaerts M, Verhaegen J: Catheter-related bacteremia due to Ewingella americana. Clin Microbiol Infect 2001, 7:103-104.

8. Pien FD, Bruce AE: Nosocomial Ewingella americana bacteremia in an intensive care unit. Arch Intern Med 1986, 146:111-112.

9. Bear N, Klugman KP, Tobiansky L, Koornhof HJ: Wound colonization by Ewingella americana. J Clin Microbiol 1986, 23:650-651. 
10. Kati C, Bibashi E, Kokolina E, Sofianou D: Case of peritonitis caused by Ewingella americana in a patient undergoing continuous ambulatory peritoneal dialysis. J Clin Microbiol 1999, 37:3733-3734

11. Hassan S, Amer S, Mittal C, Sharma R: Ewingella americana: an emerging true pathogen. Case Rep Infect Dis 2012, 2012, 730720.

doi:10.1186/1752-1947-8-86

Cite this article as: Li et al:: Peritonitis caused by Ewingella americana in a patient with peritoneal dialysis: a case report. Journal of Medical Case Reports 2014 8:86.

\section{Submit your next manuscript to BioMed Central and take full advantage of:}

- Convenient online submission

- Thorough peer review

- No space constraints or color figure charges

- Immediate publication on acceptance

- Inclusion in PubMed, CAS, Scopus and Google Scholar

- Research which is freely available for redistribution 